\title{
RACSAM
}

Rev. R. Acad. Cien. Serie A. Mat.

Vol. 103 (1), 2009, pp. 111-124

Ciencias de la Computación / Computational Sciences

\section{A technique for dynamically measuring and modifying relevance while problem solving}

\author{
Antonio Hernando and Luis de Ledesma
}

\begin{abstract}
Although there is general agreement that efficiency of problem resolution is strongly related to the problem representation adopted, computer problem solvers have been traditionally designed to keep the same representation throughout the whole of the problem solving process. A system able to change representation whilst the actual problem solving process occurs has advantages over traditional ones, not only because a representation change can improve the efficiency of problem resolution (as already proven through much research), but also because the choice of the most suitable representation may be decisively enhanced after learning about the problem during its resolution process. A natural and interesting way of performing representation changes is related to detecting irrelevant elements which can be removed out of the problem representation. In this paper, we deal formally with a new technique for assigning and changing the relevance of the elements involved in the representation during the problem resolution, on behalf of their respective importance so as to actually solve the problem.
\end{abstract}

\section{Una técnica para medir y modificar dinámicamente la relevancia en la resolución de problemas}

\begin{abstract}
Resumen. Aunque existe un consenso general sobre la fuerte dependencia entre la eficiencia en la resolución de problemas y la representación de los mismos que se adopta, los programas resolvedores de problemas tradicionalmente se han diseñado teniendo en cuenta únicamente una sola representación a lo largo de todo el proceso de resolución. Un sistema capaz de cambiar la representación mientras el mismo proceso de resolución tiene lugar ofrece ventajas respecto de los tradicionales, no sólo porque ciertos cambios de representación pueden mejorar la eficiencia del proceso de resolución (como muchas investigaciones han demostrado), sino también debido a que la tarea de seleccionar la representación más adecuada para un problema puede facilitarse decisivamente mediante el conocimiento adquirido durante el proceso de su resolución. Una manera interesante y natural de ejecutar cambios de representación se apoya en detectar la información irrelevante que puede eliminarse de la representación del problema. En el presente trabajo, presentamos formalmente una nueva técnica para asignar y modificar valores de relevancia a los elementos que integran la representación de los problemas durante el mismo proceso de resolución, de acuerdo con la importancia que tienen para resolverlos.
\end{abstract}

\section{Introduction}

Problem Solvers are computational systems able to solve some problems by simulating human problem solving performance. The choice of an adequate problem representation is no doubt an important issue

Presentado por / Submitted by Luis Laita de la Rica.

Recibido / Received: 22 de enero de 2009. Aceptado / Accepted: 4 de febrero de 2009.

Palabras clave / Keywords: Automatic Representation Changes, Problem Solver, Artificial Intelligence.

Mathematics Subject Classifications: 51A20, 68U99.

(c) 2009 Real Academia de Ciencias, España. 
in problem solving, since it is strongly related to the efficiency of the resolution process, both in human [10, 13, 14] and computer problem solving [1, 3]. Although traditional problem solvers have not undertaken the task of changing the states representation, so that the user alone is actually responsible for choosing a suitable representation beforehand [9, 11], some efforts have been done to allow the system select an adequate representation before the actual resolution of the problem starts [2]. Other approaches, involving reformulation techniques in the context of reasoning about physical systems [1], do not imply any representation change occurring during the problem solving process.

Since these latter systems only change the representation before the resolution process starts, it becomes apparent that they allow no further alteration of the problem's states space throughout the entire resolution process. Therefore, for such systems the choice of a suitable initial representation comes to be necessarily critical. In this way, the choice of an adequate representation depends on prior knowledge the system has about the problem in question; that is to say, the more knowledge the system has about the problem, the more likely may the system choose an appropriate representation. However, it may be noted that during the resolution process, the system can gain further knowledge of the problem which may eventually happen to be crucial so as to actually enabling the system select another representation which may reduce the problem states space to a significant extent, and therefore drastically improve the efficiency of the resolution process. Besides, many psychological studies on human problem solving support the evidence that humans change continuously the conception of the problem while they are solving it, by progressively acknowledging the rising importance of some problem features, as well as disposing of those regarded as irrelevant. That is to say, humans do perform a great amount of representation changes while they are solving problems [10]. Moreover, there is a specific kind of problems, namely insight problems, which are typically solved only by means of a critical representation change occurring during the actual resolution process [8, 15].

Taking all this into consideration, in this paper we will focus on demonstrating mathematically a novel technique for measuring and modifying automatically the relevance of problem features during the resolution process. A representation change consisting in dropping those irrelevant elements from the representation not only results in a new conception of the problem, but it may also involve an outstanding improvement in the efficiency of the problem's resolution. In [7] we have already studied mathematically the relationship between a given problem and the need for performing suitable representation changes. Using this technique for measuring and modifying relevance, we have so far implemented a prototype problem solver able to perform representation changes while problem solving. This technique has been successfully tested in connection to some interesting problem examples $[4,5,6]$.

In section 2, we explain our procedure of measuring a problem element's relevance. In section 3 we deal with some relevant mathematical properties about this technique. In section 4 , we summarize the main conclusions of this research.

\section{The measure of the relevance}

A computer problem solver able to perform representation changes during the resolution of a problem requires some methods for measuring the relevance of each element in the problem representation, that is to say, the importance of each element in question, so as to actually solving the problem. Therefore the need of assigning, to every feature in the problem representation, a certain relevance level, measuring its present importance in the resolution process. Obviously, relevance levels must be dynamic ones, susceptible to be modified during the whole of the resolution process.

We have designed relevance levels so that they can oscillate inside a range of values between 0 and 100 , signifying that when the relevance level of an element is 0 , this element is considered as completely irrelevant, and when its relevance level reaches the value 100, this element is considered of the utmost relevance. We will define a threshold relevance value, th, under which any representation element will be considered as irrelevant. Indeed, when the relevance value associated to an element of the representation keeps on this threshold value for some period of time, the element will be considered as irrelevant and the problem solver will eliminate it from the current representation. 
Now, we will study a procedure for changing the relevance levels during actual problem resolution. First of all, we show here some intuitive guidelines which have helped us design such procedure:

i) The higher the relevance level of an element in the problem representation is, the more this element will be used for the resolution of the problem. If an element in the problem representation is no longer used in connection to any task of problem resolution, it must be considered as irrelevant. On the contrary, if an element in the problem representation is frequently used, this must be considered as very relevant.

ii) The higher the relevance of an element in the problem representation is, the higher will become the importance of the tasks about problem resolution this element takes part in. An element is more relevant than another if the first one takes part in more relevant tasks than the second one.

The procedure adopted in this paper is based on the idea of considering two types of relevance levels associated to each element in the problem representation:

- Global Relevance Level of an element (denoted by $g$ ). It measures the importance of an element in the problem representation. It is updated periodically making use of the Recent Relevance Level associated to this element in this way:

$$
g^{\prime}=f(g, r)
$$

where $r$ stands for the recent relevance level (see next item); $g$ stands for the global relevance level the element has when just before it is updated; $f$ is a function $\mathbb{N} \times \mathbb{N} \rightarrow \mathbb{N}$, termed as 'Relevance change function' (see definition 1); and $g^{\prime}$ stands for the updated value of the global relevance level associated to this element.

- Recent Relevance Level of an element (denoted by $r$ ). It measures the importance of this element since its global relevance level was last updated. The 'Recent Relevance Level' of an element is modified in two ways:

- When the element takes part in a task with importance $t$, the recent relevance level is updated in this way:

$$
r^{\prime}=\max \{r, t\}
$$

where $r$ stands for the recent relevance level which the element has just before it is updated; $t$ stands for the importance of this element in a task; and $r^{\prime}$ stands for the updated value of the recent relevance level associated to this element.

- The 'Recent Relevance Level' of an element is also reset to value 0 each time that its 'Global Relevance Level' is updated.

In this way, a value 0 in the 'Recent Relevance Level' of an element involves that this element has not been used since the 'Global Relevance Level' has been last updated. When the 'Global Relevance Level' of an element is updated, it comes nearer to its present 'Recent Relevance Level' (as we will see in proposition 1). In this way, the 'Global Relevance Level' of an element will be continuously decreased when this element is not used for a long time

Now, we will define the 'relevance change function' over a set of parameters:

th (threshold relevance value). It must fullfil the following requirement $0 \leq t h \leq 100$. This level is established for detecting irrelevant elements. Indeed, those elements whose global relevance level is under the threshold value are regarded as irrelevant and may be consequently eliminated.

$k_{1}$ must fulfill the following requirement $1 \leq k_{1} \leq t h$. It stands for the relevance loss derived from the lack of use of an element $\left(k_{1}=g-f(g, 0)\right)$ 
$k_{2}$ must fulfill the following requirement $k_{2} \geq 1$. It is related to the increase of the updated global relevance on behalf of the recent relevance, when the latter is greater than the global relevance function.

Definition 1 (Relevance Change Function) The relevance change function, $f$ is defined as follows:

$$
f(g, r)= \begin{cases}g-\operatorname{floor}\left(\frac{g-r}{k_{2}}\right) & \text { if } g \leq r \\ \max \left(g+\text { floor }\left(\frac{k_{1} \cdot r}{g}\right)-k_{1}, \text { th }\right) & \text { if } g>r\end{cases}
$$

where floor stands for the integer part of a real number.

The relevance change function satisfies the following properties:

Proposition 1 The following holds:

i) If $r=g$, then $f(g, r)=g$

ii) If $g_{1} \geq g_{2} \geq$ th, then $f\left(g_{1}, r\right) \geq f\left(g_{2}, r\right)$

iii) If $r_{1} \geq r_{2}$, then $f\left(g, r_{1}\right) \geq f\left(g, r_{2}\right)$

iv) $\min \{g, r\} \leq f(g, r) \leq \max \{g, r\}$ for $g \geq$ th

v) If $g>$ th and $r<g$, then th $\leq f(g, r)<g$

vi) If $g>$ th and $r=0$, then $f(g, 0)=\max \left\{g-k_{1}, t h\right\}<g$

vii) If $g=$ th and $r \leq g$, then $f(g, r)=f(t h, r)=t h$

viii) If $r>g$, then $f(g, r)>g$

ix) If $g \geq t h$, then $f(g, r) \geq t h$

x) If $g \geq$ th and $r>t h$, then $f(g, r)>t h$

PROOF.

i) $f(g, g)=g-$ floor $\left((g-g) / k_{2}\right)=g$

ii) We have different cases

Case $r \leq g_{2} \leq g_{1}$

We will take the function $h(g, r)=g+f \operatorname{loor}\left(k_{1} \cdot r / g\right)-k_{1}$. In this case, the function $f(g, r)$ takes the value:

$$
f(g, r)=\max \{h(g, r), t h\}
$$

First, we will prove that $h\left(g_{2}, r\right) \leq h\left(g_{1}, r\right)$

$$
\begin{aligned}
h\left(g_{1}, r\right)-h\left(g_{2}, r\right) & =g_{1}-g_{2}+\text { floor }\left(k_{1} \cdot r / g_{1}\right)-\text { floor }\left(k_{1} \cdot r / g_{2}\right) \\
& \geq g_{1}-g_{2}+\text { floor }\left(k_{1} \cdot r / g_{1}-k_{1} \cdot r / g_{2}\right) \\
& =\left(g_{1}-g_{2}\right)+\text { floor }\left(k_{1} \cdot r \cdot\left(g_{2}-g_{1}\right) /\left(g_{1} \cdot g_{2}\right)\right)
\end{aligned}
$$

Since $0<k_{1} \leq$ th $\leq g_{2} \leq g_{1}$, and $r \leq g_{2}$ we have that:

$$
h\left(g_{1}, r\right)-h\left(g_{2}, r\right) \geq\left(g_{1}-g_{2}\right)+\left(g_{2}-g_{1}\right) \geq 0
$$

Therefore, $h\left(g_{2}, r\right) \leq h\left(g_{1}, r\right)$ 
Now, we will prove that $f\left(g_{2}, r\right) \leq f\left(g_{1}, r\right)$

If $t h \leq h\left(g_{2}, r\right)$, then, since $t h \leq h\left(g_{2}, r\right) \leq h\left(g_{1}, r\right)$ we have that

$$
f\left(g_{2}, r\right)=\max \left\{h\left(g_{2}, r\right), t h\right\}=h\left(g_{2}, r\right)
$$

Therefore, we have that $f\left(g_{2}, r\right)=h\left(g_{2}, r\right) \leq h\left(g_{1}, r\right)=f\left(g_{1}, r\right)$ If $t h>h\left(g_{2}, r\right)$ then

$$
f\left(g_{2}, r\right)=\max \left\{h\left(g_{2}, r\right), t h\right\}=t h \leq f\left(g_{1}, r\right)
$$

Case $g_{2} \leq g_{1} \leq r$

$$
\begin{aligned}
f\left(g_{1}, r\right)-f\left(g_{2}, r\right) & =g_{1}-\text { floor }\left(\left(g_{1}-r\right) / k_{2}\right)-g_{2}+\text { floor }\left(\left(g_{2}-r\right) / k_{2}\right) \\
& =\left(g_{1}-g_{2}\right)+\text { floor }\left(\left(g_{2}-r\right) / k_{2}\right)-\text { floor }\left(\left(g_{1}-r\right) / k_{2}\right) \\
& \geq\left(g_{1}-g_{2}\right)+\text { floor }\left(\left(g_{2}-r\right) / k_{2}-\left(g_{1}-r\right) / k_{2}\right) \\
& =\left(g_{1}-g_{2}\right)+\text { floor }\left(-\left(g_{1}-g_{2}\right) / k_{2}\right) \\
& \geq\left(g_{1}-g_{2}\right) \cdot\left(1+\text { floor }\left(-1 / k_{2}\right)\right)
\end{aligned}
$$

Since $k_{2} \geq 1$, we have that floor $\left(-1 / k_{2}\right)=-1$.

Consequently, $f\left(g_{1}, r\right)-f\left(g_{2}, r\right) \geq 0$.

Therefore,

$$
f\left(g_{2}, r\right) \leq f\left(g_{1}, r\right)
$$

Case $g_{2} \leq r \leq g_{1}$

Since $g_{2} \leq r \leq r$, by the first case, we have that $f\left(g_{2}, r\right) \leq f(r, r)$.

Since $r \leq r \leq g_{1}$, by the second case, we have that $f(r, r) \leq f\left(g_{1}, r\right)$.

Therefore, we have

$$
f\left(g_{2}, r\right) \leq f\left(g_{1}, r\right)
$$

iii) We have different cases:

Case $r_{2} \leq r_{1} \leq g$

$$
\begin{aligned}
f\left(g, r_{1}\right)-f\left(g, r_{2}\right) & =\text { floor }\left(k_{1} \cdot r_{1} / g\right)-\text { floor }\left(k_{1} \cdot r_{2} / g\right) \\
& \geq \text { floor }\left(k_{1} \cdot r_{1} / g-k_{1} \cdot r_{2} / g\right) \\
& \geq \text { floor }\left(k_{1} \cdot\left(r_{1}-r_{2}\right) / g\right)
\end{aligned}
$$

Since $k_{1}>0,\left(r_{1}-r_{2}\right)>0$ and $g>0$, we have that $f\left(g, r_{1}\right)-f\left(g, r_{2}\right) \geq 0$. Therefore,

$$
f\left(g, r_{1}\right) \geq f\left(g, r_{2}\right) .
$$

Case $g \leq r_{2} \leq r_{1}$

$$
\begin{aligned}
f\left(r_{1}, g\right)-f\left(r_{2}, g\right) & =\text { floor }\left(\left(g-r_{2}\right) / k_{2}\right)-\text { floor }\left(\left(g-r_{1}\right) / k_{2}\right) \\
& \geq \text { floor }\left(\left(g-r_{2}\right) / k_{2}-\left(g-r_{1}\right) / k_{2}\right) \\
& \geq \text { floor }\left(\left(r_{1}-r_{2}\right) / k_{2}\right)
\end{aligned}
$$

Since $k_{2} \geq 1$ and $\left(r_{1}-r_{2}\right)>0$ we have that $f\left(g, r_{1}\right)-f\left(g, r_{2}\right) \geq 0$. Therefore,

$$
f\left(g, r_{1}\right) \geq f\left(g, r_{2}\right) .
$$


Case $r_{2} \leq g \leq r_{1}$

Since $r_{2} \leq g \leq g$, by the first case, we have $f\left(g, r_{2}\right) \leq f(g, g)$.

Since $g \leq g \leq r_{1}$, by the second case, we have $f(g, g) \leq f\left(g, r_{1}\right)$.

Therefore,

$$
f\left(g, r_{2}\right) \leq f\left(g_{1}, r_{1}\right) .
$$

iv) By properties iii) and i), we have that

$$
\min \{g, r\}=f(\min \{g, r\}, \min \{g, r\}) \leq f(g, r) \leq f(\max \{g, r\}, \max \{g, r\})=\max \{g, r\}
$$

v) Since $k_{1} \geq 1, r \leq g-1$, and $g>0$, we have that:

$$
\text { floor }\left(k_{1} \cdot r / g\right) \leq \text { floor }\left(k_{1} \cdot(g-1) / g\right)=k_{1}+\text { floor }(-1 / g) \leq k_{1}-1<k_{1} .
$$

Therefore:

$$
f(g, r)=\max \left\{g+\text { floor }\left(k_{1} \cdot r / g\right)-k_{1}, t h\right\} \leq \max \left\{g+k_{1}-1-k_{1}, t h\right\}=\max \{g-1, t h\} .
$$

As $g-1<g$ and $t h<g$, we have that

$$
\text { th } \leq f(g, r)<g .
$$

vi) If $r=0$, then $f(g, r)=f(g, 0)=\max \left\{g+\right.$ floor $\left.\left(k_{1} \cdot 0 / g\right)-k_{1}, t h\right\}=\max \left\{g-k_{1}, t h\right\}$

vii) If $r=t h$, then $f(g, r)=f(t h, t h)=t h$

If $r<t h$, then, since $1 \leq k_{1} \leq t h$ and $r \leq t h-1$, we have that:

$$
\begin{aligned}
t h+\text { floor }(k 1 \cdot r / t h)-k_{1} & \leq t h+\text { floor }\left(k_{1} \cdot(t h-1) / t h\right)-k_{1} \\
& \leq t h+\text { floor }(-k 1 / t h) \\
& =t h-1 \\
& <t h
\end{aligned}
$$

Therefore,

$$
f(t h, r)=\max \left\{t h+\text { floor }\left(k_{1} \cdot r / t h\right)-k_{1}, t h\right\}=t h .
$$

viii) If $r>g$, then, since $(g-r) / k_{2}<0$, if $r>g$, we have that floor $\left((g-r) / k_{2}\right) \leq-1$.

Therefore, if $r>g$, then

$$
f(g, r)=g-\text { floor }\left((g-r) / k_{2}\right) \geq g+1>g .
$$

ix) We show this statement considering different cases:

If $r>g$, then, by viii), we have that $f(g, r)>g \geq t h$

If $r=g$, then, by i), we have that $f(g, r)=g \geq t h$

If $r<g$ and $g>$ th then, by v), we have that $f(g, r) \geq t h$

If $r<g$ and $g=t h$ then, by vii), we have that $f(g, r)=t h \geq t h$

x) We show this statement considering different cases:

If $g=t h$, by viii) we have that $f(g, r)>g=t h$,

If $g>t h$, by iv), we have that $f(g, r) \geq \min \{g, r\}>t h$. 


\section{Relation between use and global relevance level}

In this section we will undertake a formal study of the relation between the use made of an element and its global relevance level, by analyzing the evolution of the global relevance in elements that are used regularly. We will consider the successions $g_{n}, r_{n}$ indicating respectively the global and recent relevance levels of a element in the instant $n$ ( $n$ indicates exactly the number of global relevance updates which have been carried out). We will consider that this element is used regularly; that is to say, that it always takes part in a task with a relevance $R$ being performed periodically each $N$ updates of global relevance and the rest of time is not used. In this way,

$$
r_{n}= \begin{cases}R & \text { if } n \bmod (N+1)=0 \\ 0 & \text { if } n \bmod (N+1) \neq 0\end{cases}
$$

The succession $g_{n}$ is defined through the succession $r_{n}$ :

$$
\forall n>0 \quad g_{n}=f\left(g_{n-1}, r_{n-1}\right)
$$

By the next theorems and propositions we will show the following statements:

i) Any initial values in the Global Relevance, $g_{0}$, however distant, will in the end approach to similar levels.

ii) After a certain update, the global relevance $g_{n}$ will constantly fluctuate between some values, and these values will be under $R$.

iii) The greater $R$ and the lesser $N$ are, the greater will the global relevance level be.

iv) The greater $N$ is, the greater will fluctuations in the global relevance be.

The following proposition states that the global relevance level is always greater than the threshold relevance level, th.

Proposition 2 If $g_{0} \geq$ th, then $\forall n \in \mathbb{N} g_{n} \geq$ th.

PROOF. It is proven by induction.

Base case $n=0, g_{0} \geq t h$

Inductive case Suppose that $g_{n} \geq t h$. By ix) of proposition 1 , we have that $g_{n+1}=f\left(g_{n}, r\right) \geq t$.

The following theorem is aimed to define recursively the succession $g_{n}$ :

Theorem 1 We have that:

$$
\begin{aligned}
& \text { i) } \forall n \in \mathbb{N} g_{(n+1) \cdot(N+1)}=\max \left\{g_{n \cdot(N+1)+1}-k_{1} \cdot N, \text { th }\right\} \\
& \text { ii) } \forall m \geq 1 g_{m \cdot(N+1)}=\max \left\{f\left(g_{(m-1) \cdot(N+1)}, R\right)-k_{1} \cdot N, \text { th }\right\} \\
& \text { iii) } \forall n \in \mathbb{N} \quad \forall i \in\{1, \ldots, N+1\}, \text { if } g_{n \cdot(N+1)} \leq R \text { then } \\
& g_{n \cdot(N+1)+i}=\max \left\{g_{n \cdot(N+1)}-\operatorname{floor}\left(\left(g_{n \cdot(N+1)}-R\right) / k_{2}\right)-k_{1} \cdot(i-1), t h\right\}
\end{aligned}
$$

iv) $\forall n \in \mathbb{N} \quad \forall i \in\{1, \ldots, N+1\}$, if $g_{n \cdot(N+1)}>R$ then

$$
g_{n \cdot(N+1)+i}=\max \left\{g_{n \cdot(N+1)}+\text { floor }\left(k_{1} \cdot R / g_{n} \cdot(N+1)\right)-k_{1} \cdot i, \text { th }\right\}
$$




\section{PROOF.}

i) In case that $N=0$, we have that $g_{n+1}=\max \left\{g_{n+1}, t h\right\}=g_{n+1}$.

We will consider the case that $N>0$.

Let $n \in \mathbb{N}$ and let $i \in\{1, \ldots, N\}$.

We have that $g_{n \cdot(N+1)+i+1}=f\left(g_{n \cdot(N+1)+i}, r_{n \cdot(N+1)+i}\right)$.

Since $n \cdot(N+1)+i \bmod (N+1)=i \neq 0$, we have that:

$$
g_{n \cdot(N+1)+i+1}=f\left(g_{n \cdot(N+1)+i}, 0\right)=\max \left\{g_{n \cdot(N+1)+i}-k_{1}, t h\right\} .
$$

By applying recursively this expression $i$ times, we have that

$$
g_{n \cdot(N+1)+i+1}=\max \left\{g_{n \cdot(N+1)+1}-k_{1} \cdot i, t h\right\} .
$$

Specifically, for $i=N$, we have that

$$
g_{n \cdot(N+1)+N+1}=g_{(n+1) \cdot(N+1)}=\max \left\{g_{n \cdot(N+1)+1}-k_{1} \cdot N, t h\right\} .
$$

ii) Let $n=m-1$ and we will study the value of $g_{n \cdot(N+1)+1}$. We have these two cases:

Case $g_{n \cdot(N+1)}<R$

$$
g_{n \cdot(N+1)+1}=g_{n \cdot(N+1)}-\text { floor }\left(\left(g_{n \cdot(N+1)}-R\right) / k_{2}\right) .
$$

By i) we have that:

$$
g_{(n+1) \cdot(N+1)}=\max \left\{g_{n \cdot(N+1)}-\text { floor }\left(\left(g_{n \cdot(N+1)}-R\right) / k_{2}\right)-k_{1} \cdot N, t h\right\} .
$$

Case $g_{n \cdot(N+1)} \geq R$

$$
g_{n \cdot(N+1)+1}=\max \left\{g_{n \cdot(N+1)}+\text { floor }\left(k_{1} \cdot R / g_{n \cdot(N+1)}\right)-k_{1}, t h\right\} .
$$

By i), we have that:

$$
g_{(n+1) \cdot(N+1)}=\max \left\{g_{n \cdot(N+1)}+\text { floor }\left(k_{1} \cdot R / g_{n \cdot(N+1)}\right)-k_{1} \cdot(N+1), \text { th }\right\} .
$$

In any of these two cases, we have that

$$
g_{(n+1) \cdot(N+1)}=\max \left\{f\left(g_{n \cdot(N+1)}, R\right)-k_{1} \cdot N, t h\right\}
$$

iii) We will prove it by induction

Base case $i=1$

Since $r_{n \cdot(N+1)}=R$, we have that $g_{n \cdot(N+1)+1}=f\left(g_{n \cdot(N+1)}, R\right) \geq t h$.

Besides, we have that:

$$
\begin{aligned}
g_{n \cdot(N+1)+1} & =f\left(g_{n \cdot(N+1)}, R\right)=g_{n \cdot(N+1)}-\text { floor }\left(\left(g_{n \cdot(N+1)}-R\right) / k_{2}\right) \\
& =g_{n \cdot(N+1)}-\text { floor }\left(\left(g_{n \cdot(N+1)}-R\right) / k_{2}\right)-k_{1} \cdot(1-1) .
\end{aligned}
$$

Inductive case We will suppose that the following holds:

$g_{n \cdot(N+1)+i}=\max \left\{g_{n \cdot(N+1)}+\right.$ floor $\left(k_{1} \cdot R / g_{n \cdot(N+1)}\right)-k_{1} \cdot i$, th $\}$ where $1 \leq i<N+1$.

Since $r_{n \cdot(N+1)+i}=0$, we have that:

$$
\begin{aligned}
g_{n \cdot(N+1)+i+1} & =f\left(g_{n \cdot(N+1)+i}, 0\right) \\
& =\max \left\{g_{n \cdot(N+1)+i}-k_{1}, t h\right\} \\
& =\max \left\{g_{n \cdot(N+1)}+\text { floor }\left(k_{1} \cdot R / g_{n \cdot(N+1)}\right)-k_{1} \cdot(i+1), t h\right\}
\end{aligned}
$$


iv) Let $n \in \mathbb{N}$ and let $i \in\{1, \ldots, N+1\}$

We will prove it by induction

\section{Base case}

Since $r_{n \cdot(N+1)}=R$, we have that

$$
g_{n \cdot(N+1)+1}=f\left(g_{n \cdot(N+1)}, R\right)=\max \left\{g_{n \cdot(N+1)}+\text { floor }\left(k_{1} \cdot R / g_{n \cdot(N+1)}\right)-k_{1}, \text { th }\right\}
$$

\section{Inductive case}

$$
\begin{aligned}
& g_{n \cdot(N+1)+i}=\max \left\{g_{n \cdot(N+1)}+\operatorname{floor}\left(k_{1} \cdot R / g_{n \cdot(N+1)}\right)-k_{1} \cdot i, \text { th }\right\} \text { and } 1 \leq i<N+1 . \\
& \text { Since } r_{n \cdot(N+1)+i}=0 \\
& \qquad \begin{aligned}
g_{n \cdot(N+1)+i+1} & =f\left(g_{n \cdot(N+1)+i}, 0\right) \\
& =\max \left\{g_{n \cdot(N+1)+i}-k_{1}, t h\right\} \\
& =\max \left\{g_{n \cdot(N+1)}+\text { floor }\left(k_{1} \cdot R / g_{n \cdot(N+1)}\right)-k_{1} \cdot(i+1), t h\right\}
\end{aligned}
\end{aligned}
$$

Next, we will prove that when $n$ tends to infinite, $g_{n}$ fluctuates within a constant range. In order to prove this, we will study the evolution of $g_{n}$ in certain specific moments. First, we will define the succession, $p_{n}$, as follows:

$$
p_{n}=g_{n \cdot(N+1)} .
$$

Next, we will show that $p_{n}$ tends to a fixed point. By ii) in theorem 1, we may define $p_{n}$ recursively as follows:

$$
\begin{gathered}
p_{0}=g_{0} \\
p_{n+1}=\max \left\{f\left(p_{n}, R\right)-k_{1} \cdot N, t h\right\}
\end{gathered}
$$

In lemmas 1 and 2 we will show a relation between $p_{n}$ and $p_{n+1}$. Both these lemmas are used in theorems 2 and 3 for showing that the succession $p_{n}$ tends to a fixed value.

Lemma 1 The following holds:

i) If th $\leq p_{n} \leq R-1$, then $p_{n+1}=R$.

ii) If $p_{n}=R$, then $p_{n+1}=R$.

iii) If $p_{n} \geq R+1$, then $R \leq p_{n+1}<p_{n}$.

PROOF.

i) We have that:

$$
p_{n+1}=\max \left\{p_{n}-\text { floor }\left(\left(p_{n}-R\right) / k_{2}\right), t h\right\} \geq \max \left\{p_{n}-\text { floor }\left((R-1-R) / k_{2}\right), t h\right\}
$$

Since $k_{2} \geq 1$, we have that floor $\left(-1 / k_{2}\right)=-1$, and therefore

$$
p_{n+1} \geq \max \left\{p_{n}-\text { floor }\left(-1 / k_{2}\right), t h\right\}=\max \left\{p_{n}+1, t h\right\}
$$

Since $p_{n} \geq t h$, we have that $p_{n}+1>t h$, and therefore:

$$
p_{n+1} \geq \max \left\{p_{n}+1, t h\right\}=p_{n}+1>p_{n}
$$

Besides, we have that $p_{n+1} \leq R$. Therefore

$$
p_{n+1}=\max \left\{f\left(p_{n}, R\right), t h\right\} \leq \max \{f(R, R), R\}=R
$$


ii) We have that:

$$
p_{n+1}=\max \left\{R+\text { floor }\left(k_{1} \cdot R / R\right)-k_{1}, t h\right\}=\max \left\{R+k_{1}-k_{1}, t h\right\}=\max \{R, t h\} .
$$

Since $R>t h$, we have that

$$
p_{n+1}=R
$$

iii) We have that:

$$
\begin{aligned}
p_{n+1} & =\max \left\{p_{n}+\operatorname{floor}\left(k_{1} \cdot R / p_{n}\right)-k_{1}, t h\right\} \\
& \leq \max \left\{p_{n}+\text { floor }\left(k_{1} \cdot\left(p_{n}-1\right) / p_{n}\right)-k_{1}, t h\right\} \\
& \leq \max \left\{p_{n}+k_{1}+\text { floor }\left(-k_{1} / p_{n}\right)-k_{1}, t h\right\} \\
& \leq \max \left\{p_{n}+\text { floor }(-k 1 / p n), t h\right\} .
\end{aligned}
$$

Since $k_{1} \leq t h \leq p_{n}$ and floor $\left(-k_{1} / p_{n}\right)=-1$, we have that

$$
p_{n+1} \leq \max \left\{p_{n}-1, t h\right\} .
$$

Since $t h<R$ and $R<p_{n}$, we have that $t h \leq p_{n}-1$ and therefore

$$
p_{n+1} \leq \max \left\{p_{n}-1, t h\right\}=p_{n}-1<p_{n} .
$$

Besides, we have that $p_{n+1} \geq R$

$$
p_{n+1}=\max \left\{f\left(p_{n}, R\right), t h\right\} \geq \max \{f(R, R), t h\}=R .
$$

Theorem 2 If $N=0$, then we have that:

$$
\exists n \in \mathbb{N} \quad \forall m \geq n \quad p_{m}=R
$$

PROOF. We will prove that $\exists n \geq 0$ such that $p_{n}=R$. Once we have proved it, we will prove this lemma by applying ii)in lemma 1 . We will consider these cases:

Case $t h<p_{0} \leq R-1$

By i) in lemma 1 , we have that $p_{1}=R$. By ii) in lemma 1 , we have that

$$
\forall m \geq 1 \quad p_{m}=R
$$

Case $p_{0}=R$

By ii) in lemma 1, we have that

$$
\forall m \geq 0 \quad p_{m}=R
$$

Case $p_{0} \geq R+1$

By iii) in lemma 1 we have that:

$$
\exists n \geq 0 \text { such that } \quad p_{n}=R
$$

Therefore, by ii) in lemma 1 , we have that

$$
\forall m \geq n \quad p_{m}=R
$$


Lemma 2 If $N \geq 1$, then we have that:

i) If $p_{n}<\max \left\{R-k_{1} \cdot k_{2} \cdot N-1\right.$, th $\}$ then

$$
p_{n}<p_{n+1} \leq \max \left\{R-k_{1} \cdot k_{2} \cdot N, t h\right\}
$$

ii) If $p_{n}>\max \left\{R-k_{1} \cdot k_{2} \cdot N+k_{2}-1\right.$, th $\}$ and th $\leq p_{n} \leq R$, then

$$
\max \left\{R-k_{1} \cdot k_{2} \cdot N+k_{2}-1, t h\right\} \leq p_{n+1}<p_{n}
$$

iii) If $p_{n}>\max \left\{R-k_{1} \cdot k_{2} \cdot N+k_{2}-1\right.$, th $\}$ and $p_{n} \geq R+1$, then

$$
\max \left\{R-k_{1} \cdot k_{2} \cdot N+k_{2}-1, t h\right\} \leq p_{n+1}<p_{n}
$$

iv) If $p_{n}=\max \left\{R-k_{1} \cdot k_{2} \cdot N+i\right.$, th $\}$ where $0 \leq i \leq k_{2}-1$, then

$$
p_{n+1}=p_{n}
$$

Proof.

i) Since $p_{n} \leq \max \left\{R-k_{1} \cdot k_{2} \cdot N-1\right.$, th $\}$, we have that $p_{n} \leq R-k_{1} \cdot k_{2} \cdot N-1<R$

We will prove that $p_{n+1}>p_{n}$.

Since we have that $p_{n}<R$.

$$
\begin{aligned}
p_{n+1} & =\max \left\{f\left(p_{n}, R\right)-k_{1} \cdot N, t h\right\} \\
& =\max \left\{p_{n}-\text { floor }\left(\left(p_{n}-R\right) / k_{2}\right)-k_{1} \cdot N, t h\right\} \\
& \geq \max \left\{p_{n}-\text { floor }\left(\left(R-k_{1} \cdot k_{2} \cdot N-1-R\right) / k_{2}\right)-k_{1} \cdot N, t h\right\} \\
& \geq \max \left\{p n+k_{1} \cdot N-\text { floor }(-1 / k 2)-k_{1} \cdot N, t h\right\} \\
& =\max \left\{p_{n}-\text { floor }\left(-1 / k_{2}\right), t h\right\} .
\end{aligned}
$$

Since $k_{2} \geq 1$, we have that floor $\left(-1 / k_{2}\right)=-1$. Therefore

$$
p_{n+1} \geq \max \left\{p_{n}-\text { floor }\left(-1 / k_{2}\right), t h\right\}=\max \left\{p_{n}+1, t h\right\} .
$$

Since $p_{n} \geq t h$, we have that $p_{n}+1>t h$. Therefore: $p_{n+1} \geq \max \left\{p_{n}+1, t h\right\}=p_{n}+1>p_{n}$. We will prove that $p_{n+1} \leq \max \left\{R-k_{1} \cdot k_{2} \cdot N\right.$, th $\}$.

$$
p_{n+1}=\max \left\{f\left(p_{n}, R\right)-k_{1} \cdot N, t h\right\} \leq \max \left\{f\left(R-k_{1} \cdot k_{2} \cdot N-1, R\right)-k_{1} \cdot N, t h\right\}
$$

Since $R-k_{1} \cdot k_{2} \cdot N-1<R$, we have that

$$
\begin{aligned}
p_{n+1} & \leq \max \left\{R-k_{1} \cdot k_{2} \cdot N-1-\text { floor }\left(\left(R-k_{1} \cdot k_{2} \cdot N-1-R\right) / k_{2}\right)-k_{1} \cdot N, t h\right\} \\
& \leq \max \left\{R-k_{1} \cdot k_{2} \cdot N-1+k_{1} \cdot N+1-k_{1} \cdot N, t h\right\} \\
& =\max \left\{R-k_{1} \cdot k_{2} \cdot N, t h\right\}
\end{aligned}
$$

ii) We will prove that $p_{n+1}<p_{n}$.

Since $p_{n} \leq R$, we have that:

$$
p_{n+1}=\max \left\{f\left(p_{n}, R\right)-k_{1} \cdot N, t h\right\}=\max \left\{p_{n}-\text { floor }\left(\left(p_{n}-R\right) / k_{2}\right)-k_{1} \cdot N, t h\right\} .
$$

Since $p_{n} \geq R-k_{1} \cdot k_{2} \cdot N+k_{2}$, we have that:

$$
p_{n+1} \leq \max \left\{p_{n}-\text { floor }\left(\left(R-k_{1} \cdot k_{2} \cdot N+k_{2}-R\right) / k_{2}\right)-k_{1} \cdot N, t h\right\}=\max \left\{p_{n}-1, t h\right\} .
$$

Since $p_{n}>t h$, we have that $t h \leq p_{n}-1$. Therefore:

$$
p_{n+1} \leq \max \left\{p_{n}-1, t h\right\}=p_{n}-1<p_{n}
$$


We will prove that $p_{n+1} \geq \max \left\{R-k_{1} \cdot k_{2} \cdot N+k_{2}-1, t h\right\}$

Since $R-k_{1} \cdot k_{2} \cdot N+k_{2} \leq p_{n} \leq R$, we have that

$$
\begin{aligned}
p_{n+1} & =\max \left\{f\left(p_{n}, R\right)-k_{1} \cdot N, t h\right\} \\
& \geq \max \left\{f\left(R-k_{1} \cdot k_{2} \cdot N+k_{2}, R\right)-k_{1} \cdot N, t h\right\} \\
& \geq \max \left\{R-k_{1} \cdot k_{2} \cdot N+k_{2}-\text { floor }\left(\left(R-k_{1} \cdot k_{2} \cdot N+k_{2}-R\right) / k_{2}\right)-k_{1} \cdot N, t h\right\} \\
& \geq \max \left\{R-k_{1} \cdot k_{2} \cdot N+k_{2}+k_{1} \cdot N-1-1 \cdot N, t h\right\} \\
& =\max \left\{R-k_{1} \cdot k_{2} \cdot N+k_{2}-1, t h\right\}
\end{aligned}
$$

iii) We will prove that $p_{n+1}<p_{n}$.

Since $p_{n}>R$, we have that:

$$
\begin{aligned}
p_{n+1} & =\max \left\{f\left(p_{n}, R\right)-k_{1} \cdot N, \text { th }\right\} \\
& =\max \left\{p_{n}+\text { floor }\left(k_{1} \cdot R / p_{n}\right)-k_{1} \cdot(N+1), t h\right\} \\
& \leq \max \left\{p_{n}+\text { floor }\left(k_{1} \cdot p_{n} / p_{n}\right)-k_{1} \cdot(N+1), t h\right\} \\
& =\max \left\{p_{n}-k_{1} \cdot N, t h\right\} \leq \max \left\{p_{n}-1, t h\right\} .
\end{aligned}
$$

Since $t h<R+1 \leq p_{n}$, we have that $t h \leq p_{n}-1$

$$
p_{n+1} \leq \max \left\{p_{n}-1, t h\right\}=p_{n}-1<p_{n}
$$

We will prove that $p_{n+1} \geq \max \left\{R-k_{1} \cdot k_{2} \cdot N+k_{2}-1, t h\right\}$.

Since $p_{n} \geq R-k_{1} \cdot k_{2} \cdot N+k_{2}$, we have that:

$$
\begin{aligned}
p_{n+1} & =\max \left\{f\left(p_{n}, R\right)-k_{1} \cdot N, t h\right\} \\
& =\max \left\{p_{n}+\text { floor }\left(k_{1} \cdot R / p_{n}\right)-k_{1} \cdot(N+1), t h\right\} \\
& \geq \max \left\{f\left(R-k_{1} \cdot k_{2} \cdot N+k_{2}, R\right)-k_{1} \cdot N, t h\right\}
\end{aligned}
$$

Since $k_{1} \geq 1$ and $N \geq 1$, we have that $R-k_{1} \cdot k_{2} \cdot N+k_{2} \leq R-k_{2}+k_{2}=R$

$$
\begin{aligned}
p_{n+1} & \geq \max \left\{R-k_{1} \cdot k_{2} \cdot N+k_{2}-\text { floor }\left(\left(R-k_{1} \cdot k_{2} \cdot N+k_{2}-R\right) / k_{2}\right)-k_{1} \cdot N, t h\right\} \\
& \geq \max \left\{R-k_{1} \cdot k_{2} \cdot N+k_{2}+k_{1} \cdot N-1-k_{1} \cdot N, t h\right\} \\
& =\max \left\{R-k_{1} \cdot k_{2} \cdot N+k_{2}-1, t h\right\} .
\end{aligned}
$$

iv) We will consider these two cases:

Case $p_{n}=R-k_{1} \cdot k_{2} \cdot N+i \geq t h$

Since $p_{n} \leq R-k_{1} \cdot k_{2} \cdot N+k_{2}-1 \leq R-k_{2}+k_{2}-1=R-1<R$, we have that:

$$
\begin{aligned}
p_{n+1} & =\max \left\{p_{n}-\text { floor }\left(\left(p_{n}-R\right) / k_{2}\right)-k_{1} \cdot N, t h\right\} \\
& =\max \left\{p_{n}-\text { floor }\left(\left(R-k_{1} \cdot k_{2} \cdot N+i-R\right) / k_{2}\right)-k_{1} \cdot N, t h\right\} \\
& =\max \left\{p_{n}-\text { floor }\left(i / k_{2}\right), t h\right\}
\end{aligned}
$$

Since $0 \leq i \leq k_{2}-1$, we have that floor $\left(i / k_{2}\right)=0$, and consequently:

$$
p_{n+1}=\max \left\{p_{n}-\text { floor }\left(\left(i / k_{2}\right), t h\right\}=\max \left\{p_{n}, t h\right\}=p_{n}\right.
$$

Case $p_{n}=t h \geq R-k_{1} \cdot k_{2} \cdot N+i$

We have that:

$$
\begin{aligned}
& \text { th }- \text { floor }\left((t h-R) / k_{2}\right)-k_{1} \cdot N \leq t h-\text { floor }\left(\left(R-k_{1} \cdot k_{2} \cdot N+i-R\right) / k_{2}\right)-k_{1} \cdot N \\
& \quad \leq t h+k_{1} \cdot N-\text { floor }\left(i / k_{2}\right)-k_{1} \cdot N \\
& \quad=t h
\end{aligned}
$$


Therefore,

$$
p_{n+1}=\max \left\{t h-\text { floor }\left((t h-R) / k_{2}\right)-k_{1} \cdot N, t h\right\}=t h=p_{n} .
$$

Theorem 3 If $N \geq 1$, we have that:
i) If $p_{0} \leq \max \left\{R-k_{1} \cdot k_{2} \cdot N\right.$, th $\}$, then $\exists n \geq 0 \forall m \geq n \quad p_{m}=\max \left\{R-k_{1} \cdot k_{2} \cdot N\right.$, th $\}$.
ii) If $p_{0} \geq \max \left\{R-k_{1} \cdot k_{2} \cdot N+k_{2}-1\right.$, th $\}$, then $\exists n \geq 0 \forall m \geq n \quad p_{m}=\max \left\{R-k_{1} \cdot k_{2} \cdot N+k_{2}-1\right.$, th $\}$
iii) If $\max \left\{R-k_{1} \cdot k_{2} \cdot N+k_{2}-1\right.$, th $\} \leq p_{0} \leq \max \left\{R-k_{1} \cdot k_{2} \cdot N\right.$, th $\}$, then $\forall m \geq 0 \quad p_{m}=p_{0}$.

PROOF.

i) By taking into account i) in lemma 2, $\exists n>0 \quad p_{n}=\max \left\{R-k_{1} \cdot k_{2} \cdot N\right.$, th $\}$. By taking into account iv) in lemma $2, \forall m>n \quad p_{m}=p_{n}$.

ii) By taking into account ii) in lemma 2, $\exists n>0 \quad p_{n}=\max \left\{R-k_{1} \cdot k_{2} \cdot N+k_{2}-1\right.$, th $\}$. By taking into account iv) in lemma $2, \forall m>n \quad p_{m}=p_{n}$.

iii) By taking into account iv) in lemma 2, $\forall m \geq 0 \quad p_{m}=p_{0}$.

\section{Conclusions}

As has been seen, assigning relevance values to the various elements shaping a problem representation is really useful in order to solve it. This paper describes some new techniques for establishing and modifying such relevance values within the performance of a computer problem solver we have implemented and which, unlike its predecessors, is able to execute crucial changes in the problem representation in virtually any moment of the solving process. These representation changes occur as an autonomous fulfillment of our problem solver, with no need for the user to order or activate them, being related to the measure of relevance values in the way described in this paper. Thus, automatic representation changes within the system, dependent on relevance values of the problem elements, offer a valid simulation of unconscious human behavior while problem solving. Further specific details about the architecture and performance of our problem solver are to be found in some of the references below.

\section{References}

[1] Choueiry, B. Y., Iwasaki, Y. And McIlraith, S., (2005). Towards a practical theory of reformulation for reasoning about physical systems, Artificial Intelligence, 162, 145-204.

[2] FInK, E., (2003). Changes of Problem Representation: Theory and Experiments, Springer, Berlin.

[3] Gentner, D. And Stevens, A. L., (1983). Mental Models, Lawrence Erlbaum Associates, Mahwah (NJ).

[4] Hernando, A., De Ledesma, L. And Laita, L. M., (2003). Toward an AI Theory of Insight, in: Proc. 12th IASTED Internat. Conf. on Applied Simulation and Modelling (Marbella), Anaheim, ACTA Press, 181-186.

[5] Hernando, A., De Ledesma, L. And Laita, L. M., (2005). A Programme embodying Insight and Representation Changes in Problem Solving, in: Proc. 9th World Multi-Conference on Systemics, Cybernetics and Informatics, Orlando, International Institute of Informatics and Systemics, 58-63. 
[6] Hernando, A., De Ledesma, L. And Laita, L. M., (2007). An Approach to Representation Changes While Executing Problem Solver Intelligent Systems, in: Proc. 6th IEEE Internat. Conf. on Cognitive Informatics, (Lake Tahoe), IEEE Computer Society, 35-42.

[7] Hernando, A., De Ledesma, L. And Laita, L. M., (2008). A System Simulating Representation Change Phenomena While Problem Solving, Mathematics and Computers in Simulation, 78, 89-106.

[8] Kaplan, C. A. And Simon, H. A., (1990). In Search of Insight, Cognitive Psychology, 22, 374-419.

[9] Laird, J. E., Newell, A. And Rosenbloom, P. S., (1987). SOAR: An Architecture for General Intelligence, Artificial Intelligence, 33, (1), 1-64.

[10] Newell, A. And Simon, H. A., (1972). Human Problem Solving, Prentice-Hall, Englewood Cliffs.

[11] Newell, A., (1992). Unified theories of cognition and the role of SOAR, in: J. A. Michon, A. Akyrek (Eds.), SOAR: A Cognitive Architecture in Perspective, Kluwer Academic Publishers, Boston (MA), 25-79.

[12] Polya, G., (1957). How to Solve It, Doubleday, Garden City (NY).

[13] QIn, Y. And Simon, H. A., (1992). Imaginary and mental models in problem solving, in: Proc. AAAI 1992 Spring Symposium on Reasoning with Diagramatic Representation, Stanford University, Palo Alto (CA).

[14] Simon, H. A., (1979). Models of Thought, Yale University Press, New Haven.

[15] Simon, H. A., (1995). Explaining the Ineffable: AI on the topics of Intuition, Insight and Inspiration, in: Proc. 14th International Joint Conference on Artificial Intelligence, Montreal, 939-948.

\section{Antonio Hernando}

Profesor Titular de Universidad Interino

Dept. Sistemas Inteligentes Aplicados

Escuela Unversitaria de Informática

Universidad Politécnica de Madrid

\section{Luis de Ledesma}

Catedrático de Universidad

Dept. Inteligencia Artificial

Facultad de Informática

Universidad Politécnica de Madrid 